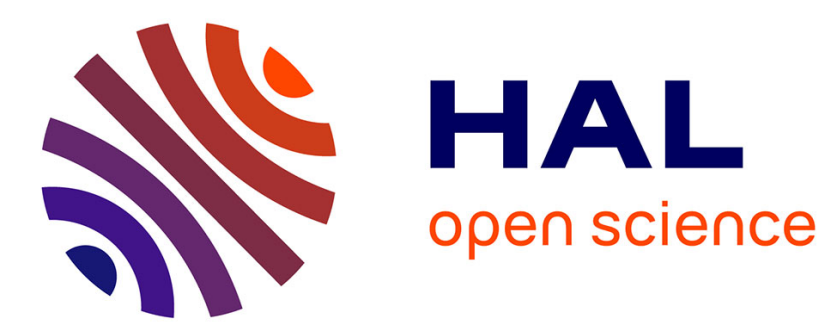

\title{
Allergie de contact aux huiles essentielles
}

M. Avenel-Audran

\section{To cite this version:}

M. Avenel-Audran. Allergie de contact aux huiles essentielles. Revue francaise d'allergologie, 2019, 59, pp.216 - 218. 10.1016/j.reval.2019.02.209 . hal-03486331

\section{HAL Id: hal-03486331 \\ https://hal.science/hal-03486331}

Submitted on 20 Dec 2021

HAL is a multi-disciplinary open access archive for the deposit and dissemination of scientific research documents, whether they are published or not. The documents may come from teaching and research institutions in France or abroad, or from public or private research centers.
L'archive ouverte pluridisciplinaire $\mathbf{H A L}$, est destinée au dépôt et à la diffusion de documents scientifiques de niveau recherche, publiés ou non, émanant des établissements d'enseignement et de recherche français ou étrangers, des laboratoires publics ou privés.

\section{다)(1) $(5$}

Distributed under a Creative Commons Attribution - NonCommercial| 4.0 International 
Version of Record: https://www.sciencedirect.com/science/article/pii/S187703201930243X

Manuscript_9f1bd79d3f60eac54af0ff6db9ebf3c4

\title{
Allergie de contact aux huiles essentielles
}

Allergic contact dermatitis from essential oils

\author{
Martine Avenel-Audran \\ Service de Dermatologie - CHU Angers \\ martine.audran@gmail.com
}


Mots clés : eczéma de contact, huiles essentielles, essence de lavande, tea tree oil, plantes

Keywords: contact eczema, essential oils, lavender essential oil, tea tree oil, plants

Surfant sur la grande vague du " bio » et du " naturel », les huiles essentielles sont partout. L'aromathérapie est très prisée, les cosmétiques utilisent leur côté aromatique et leur présence est aussi un argument de vente pour des produits ménagers « bio » de plus en plus nombreux et variés.

\section{Qu'est-ce qu'une huile essentielle?}

L'huile essentielle (HE), appelée aussi essence, est un produit aromatique obtenu à partir d'une plante, fraîche le plus souvent, qui répond à la norme ISO 9235. Toutes les parties de la plante sont concernées et différentes techniques sont utilisées. La plus courante est la distillation à la vapeur, hydrodistillation ou entraînement à la vapeur. La distillation sèche est aussi utilisée pour les pétales et les écorces. L'extraction mécanique du péricarpe permet des récolter les essences d'agrumes.

L'HE ne doit pas être confondue avec d'autres extraits de plantes utilisés dans les parfums, comme les concrètes qui ont des extraits de plantes fraîches (pétales de fleurs surtout) obtenus par solvants, les résinoïdes qui sont des extraits de plantes sèches obtenus aussi par solvants, les absolues qui sont des extractions par l'éthanol de concrètes ou de résinoödes et enfin les hydrolats, résidus aqueux de distillation qui contiennent des restes d'huiles essentielles. Il est important aussi de ne pas confondre HE et huiles végétales, qui sont, elles, obtenues par pression de différentes parties des plantes, graines le plus souvent. Certaines sont inoffensives telles l'huile de tournesol ou l'huile d'olive, mais d'autres comme l'huile de nigelle par exemple peuvent occasionner des accidents cutanés parfois sévères.

La composition des HEs est complexe, et très variable selon divers paramètres tels que l'espèce, la partie de la plante, le biotope, la provenance, le fabricant... C'est pourquoi seules les HEs chémotypées (présence des principaux composants vérifiée par chromatographie) sont recommandées pour l'aromathérapie. Les composants peuvent être très nombreux, entre 100 et 250 voire plus jusqu'à plus de 400, et souvent communs à plusieurs HEs. Le groupe le plus important est celui des dérivés terpéniques, dont le pouvoir allergisant est facilité par une sensibilité particulière à l'oxydation et au soleil. Les plus souvent présents 
sont le limonène et le béta-caryophyllène. Le linalol, l'alpha-pinène, l'eucalyptol, le géraniol, la carvone et bien d'autres appartiennent à ce groupe. Les phénylpropanoïdes sont la deuxième famille de composants à laquelle appartiennent par exemple, alcool et aldéhyde cinnamiques, eugénol, coumarine, allergènes bien connus en allergie de contact. Enfin peuvent de retrouver en très faible quantité des dérivés d'acides gras, de terpènes ou d'acides aminés, voire des pesticides (en dehors de l'agriculture biologique)

\section{Quelques huiles essentielles souvent responsables d'allergie de contact}

D'après De Groot (1,2), 79 HEs ont été reconnues responsables d'allergies de contact dans la littérature. Celle qui occasionne le plus de publications est l'HE de l'arbre à thé (tea tree oil) extraite par distillation des branches et feuilles de Melaleuca alternifolia, une myrtacée qui pousse naturellement en Australie. Déjà utilisée par les arborigènes, elle est maintenant mondialement réputée pour ses nombreuses vertus médicinales. Elle serait un « remède naturel » contre toutes les infections cutanées (en particulier fongiques), l'acné, le psoriasis, les ulcères de jambes et même les dermites de contact... Elle entre aussi dans la composition d'autres produits " naturels » tels que cosmétiques ou produits ménagers. Parmi ses 220 composants identifiés, on trouve l'alpha-pinène en commun entre autres avec les essences de lavande, de menthe et de térébenthine, ainsi que le 1-8-cinéol (eucalyptol) aussi en commun avec l'eucalyptus. Le premier cas d'eczéma de contact a été publié en Australie en 1991, et d'autres ont été publiés depuis en particulier des formes sévères d'eczémas systémiques ou à type d'érythème polymorphe. La tea tree oil a été ajoutée depuis 2004 à la batterie standard australienne et donne des tests positifs chez $2,5 \%$ des patients testés. La prévalence est plus faible aux USA $(1,4 \%)$ et en Europe $(0,9 \%)$. Le risque de sensibilisation est d'autant plus élevé que la concentration est élevée, et il est recommandé de ne pas utiliser sur la peau une concentration supérieure à $10 \%$. C'est d'ailleurs la concentration du patch-test utilisée en Australie, alors qu'en Europe elle est de 5\%. L'HE d'eucalyptus globuleux, arbre proche de la famille des Myrtacées contient aussi eucalyptol et alphapinène. Elle est très utilisée en aromathérapie comme anti-infectieux et décongestionnant et aussi dans les produits ménagers. Quelques cas d'eczéma de contact ont été rapportés. L'autre grande famille de plantes aromatiques d'où sont extraites des HEs est la famille des Lamiacées. L'HE de lavande fine (Lavandula augustifolia), obtenue par hydrodistillation des fleurs, est l'HE la plus utilisée en France. Elle est réputée pour ses multiples vertus thérapeutiques, sédative, anti-dépressive, antimicrobienne, antifongique, cicatrisante... Elle 
est aussi un excipient de topiques médicamenteux et très largement utilisée dans les parfums, en particulier masculins. Parmi les 450 composants identifiés, on retrouve le linalol, l'alpha-pinène, l'eucalyptol et le béta-caryophyllène. Une quarantaine de publications font état d'allergie de contact à l'essence de lavande. La prévalence dans la population de patients testés en routine varie de $0,2 \%$ à $1,2 \%$ mais elle s'élève à $30 \%$ dans la population des patients allergiques aux parfums. Cet eczéma se voit chez des professionnels comme les aromathérapeutes et les kinésithérapeutes qui utilisent des huiles de massage qui en contiennent, après usage de cosmétiques ou de topiques médicamenteux, ou encore est dû à la goutte d'essence pure déposée le soir sur l'oreiller pour faciliter le sommeil. Elle se teste habituellement à $2 \%$ dans la vaseline.

Des menthes, autres Lamiacées, sont aussi extraites des HEs à risque allergique. L'HE de menthe commune ou spearmint (Mentha spicata) compte 250 composants dont limonène, eucalyptol, béta-caryophyllène, menthol et carvone. C'est ce dernier qui est l'agent responsable le plus fréquent des cas d'eczémas de contact, en particulier avec les dentifrices. Elle se teste à $2 \%$ dans la vaseline. L'HE de menthe poivrée ou peppermint (Mentha piperata) comporte plus de 330 composants dont le menthol est le principal, mais aussi limonène et eucalyptol. Elle a un usage médicinal, à la fois comme principe actif et comme excipient, mais aussi cosmétique et comme arôme (dentifrice, aliments, tabac). Plus de 40 publications rapportent un eczéma de contact due à cette essence. Elle se teste à $2 \%$ dans la vaseline.

L'HE de laurier noble extraite de Laurus Nobilis de la famille des Lauracées contient des lactones sequiterpèniques sensibilisantes, mais aussi du linalol, de l'eucalyptol et de l'eugénol est bien connue pour son risque sensibilisant. Une vingtaine de cas d'eczéma de contact ont été rapportés avec l'HE de cannelle (Cinnamomum verum) de la même famille, qui contient eugénol, linalol, beta-caryophyllène et aldéhyde cinnamique et est utilisée contre les verrues ou dans les dentifrices.

Les autres HEs les plus souvent responsables d'allergie de contact sont les HEs de géranium, térébenthine, ylang ylang, cananga, citron, jasmin, rose, citronelle, girofle...

La fréquence des allergies à plusieurs huiles essentielles ainsi qu'aux marqueurs des allergies aux parfums (FMI, FMII, Myroxylon pereirae) est extrêmement fréquente. Ceci est en partie lié à la présence de composants communs. C'est le cas, par exemple, des peroxydes de linalol et de limonène. Ces marqueurs sont maintenant entrés dans la batterie standard 
européenne, en raison de la fréquence de leur positivité quand on les teste. (Tableau I). II reste cependant indispensable de tester l'huile essentielle utilisée en respectant sa concentration de testage, la tester pure conduit au risque d'irritation ou de réaction allergique très violente. Une batterie complémentaire "huiles essentielles » peut aussi s'avérer utile pour dépister les co-allergies.

En conclusion, les HEs sont des produits d'origine naturelle, à usage cutané prédominant, de composition complexe, de plus en plus prisés. A ce mode d'application s'associe une augmentation de l'utilisation. Bon nombre des composants sont à risque d'allergie de contact et les co-allergies entre HEs et parfums sont fréquents. Un respect des règles d'utilisation et particulièrement de concentration d'usage est indispensable pour éviter la multiplication des cas d'eczémas de contact. 


\section{Références}

1-De Groot AC, Schmidt E. Essentials oils. CRC Press Taylor \& Francis Group, Boca Raton, 2016; 1044 pages

2- De Groot AC, Schmidt E. Essential Oils, Dermatitis 2016; 27

Part I: Introduction: 39-42,

Part II: General Aspects: 43-9,

Part III: Chemical composition: 161-9

Part IV: Contact Allergy: 170-5

Part V: Peppermint Oil, Lavender Oil, and Lemongrass Oil: 325-332

Tea tree oil: contact allergy and chemical composition: 129-43 
Tableau I

D’après Rudbäck J et al Contact Dermatitis 2015; 73:253-4

\begin{tabular}{|c|c|c|}
\hline Huile essentielle & Limonène & Linalol \\
\hline Géranium & + & + \\
\hline Jasmin & - & + \\
\hline Lavande & + & + \\
\hline Orange amère & + & + \\
\hline Pin & + & - \\
\hline Ylang Ylang & - & + \\
\hline Tea tree & + & + \\
\hline Laurier & + & + \\
\hline Menthe commune & + & + \\
\hline
\end{tabular}

